\title{
60 years of IAU symposia
}

\author{
Areg M. Mickaelian \\ Byurakan Astrophysical Observatory (BAO), Armenia \\ E-mail: aregmick@yahoo.com
}

The history of the International Astronomical Union (IAU) meetings goes back to 1922 when the IAU I General Assembly (GA) was held in Rome, Italy, following the IAU creation in 1919. However, until 1953, no individual symposia were organized and GA was the only official gathering for astronomers. Altogether, 8 IAU GA were held during 1922-1952.

The IAU Symposium 1 on "Co-ordination of Galactic Research" was held during June 17-22, 1953 in Groningen, Netherlands followed by another one (IAU Symposium 2: "Gas Dynamics of Cosmic Clouds") on July 6-11 in Cambridge, UK. Starting with 1955, regularly several IAU symposia were held in different places, as well as since 1959 IAU began to also organize colloquia to discuss relatively smaller topics. 20 IAU colloquia numbered as I-XX were held in 1959-1971, and another series of IAU colloquia was organized in 1968-2005, numbered as Nos. 1-200. At present IAU symposia are the only official scientific meetings, 9 of them organized every year. IAU S304 "Multiwavelength AGN Surveys and Studies" held in Armenia was the last one in 2013. Thus, IAU has a 60 -year history of symposia and altogether 304 such meetings have been held, in average 5 ones annually.

At present most of the IAU symposia during the years of GA are being organized in frame of the GA, typically 6 symposia during each GA. This was the case during the GA in 1994 (Hague, Netherlands), 1997 (Kyoto, Japan), 2000 (Manchester, UK), 2003 (Sydney, Australia), 2006 (Prague, Czech Republic) and 2009 (Rio de Janeiro, Brazil). 8 IAU symposia were held during the GA XXVIII in Beijing (2012). Altogether, 29 IAU GA have been organized during 1922-2012, including 28 regular ones and 1 Extraordinary (1973 in Warsaw, Poland), typically once per 3 years.

Since 1974, IAU also organizes regional meetings in Europe, Asia and Pacific (APRIM, Asia-Pacific Regional IAU Meeting), Latin America (LARIM, Latin-American Regional IAU Meeting), and Middle East and Africa (MEARIM, Middle East and African Regional IAU Meeting). The European ones were discontinued in 1990 after the creation of the European Astronomical Union (EAS) and organization of the yearly JENAMs (at present EWASS - European Week of Astronomy and Space Science). The three others, APRIM (since 1978), LARIM (since 1978), and MEARIM (since 2008) are being organized every 2 or 3 years. Altogether 38 regional meetings have been organized.

As mentioned, 304 IAU symposia have been held on various topics of astronomy and astrophysics. The most often subjects were:

- various matters related to Stars (37)

- various Astrophysical Processes (28)

- Galaxies (27)

- Cosmology (17)

- Sun (17, including Solar Activity 7)

- Solar System (16)

- Star Formation and Evolution (15) 

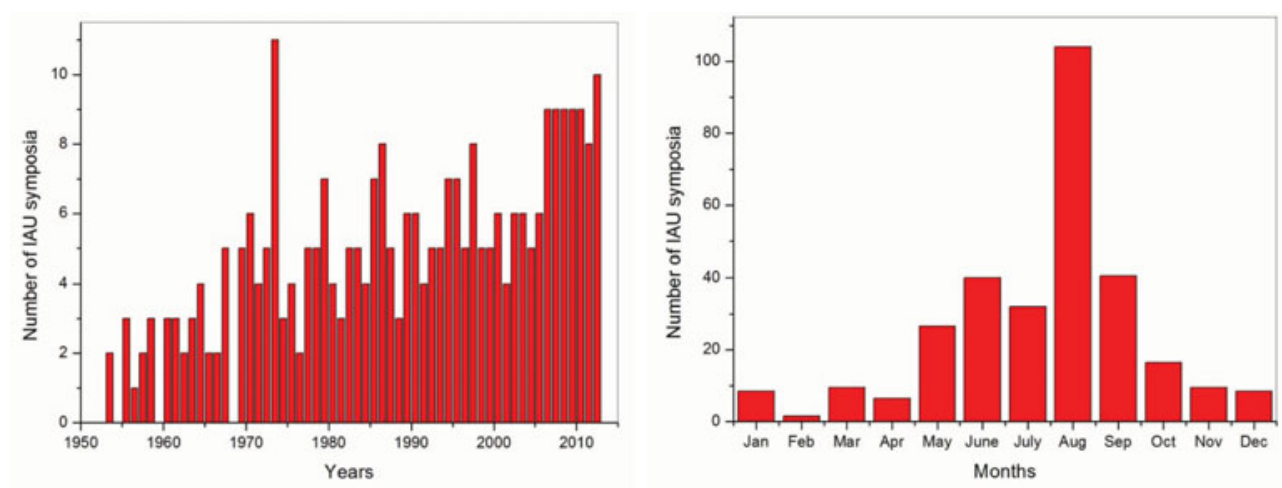

Figure 1. Distribution of the number of IAU symposia by years (left) and by months (right).

- Instrumentation and Methods (14)

- Multiwavelength Astronomy ( $\gamma$-ray, X-ray, UV, IR and Radio; 13)

- AGN (10)

- Exoplanets and SETI (9)

- Our Galaxy (9)

- Planetary Nebulae (9)

- Astrochemistry (8)

- Binary Stars (8)

- Interstellar Medium (8)

- Astrometry (7)

- Earth (7)

- Fundamental Astronomy (6)

- Magnetic Fields (6)

- Star Clusters (6)

- Magelanic Clouds (5)

- Neutron Stars and Pulsars (5)

- Wolf-Rayet Stars (5)

- Supernovae (4)

- Astronomical Surveys (4)

The distribution of the IAU symposia by years and by months is given in Figure 1 . An increase in numbers is clearly seen in case of the distribution by years. Most often (104) IAU symposia have been held in August, mainly connected to the IAU GA. Only 1 symposium (and a part of another one) has been held in February.

The 304 meetings have been organized in 43 countries; most often in the USA (42), France (23), Australia (20), China (18), Italy (18), UK (18), Germany (14), Netherlands (14), Brazil (13), Japan (13), Canada (9), Switzerland (9), Russia (8), Spain (8), Argentina (6), Czech Republic (6), Poland (6), Sweden (6), Armenia (5), and India (5). Altogether, 171 cities and towns have been hosts for the IAU symposia, most often Paris (12), Beijing (10), Sydney (9), Rio de Janeiro (8), Kyoto (7), Prague (7), The Hague (6), Geneve (5), St. Petersburg (5), Manchester (5), Byurakan (4), Cambridge, MA (4), Canberra (4), Heidelberg (4), and Tokyo (4). 